Magdalena Knetki-Wróblewska', Dariusz M. Kowalski', Grzegorz Czyżewicz², Maciej Bryl', Anna Wrona ${ }^{4}$, Rafał Dziadziuszko ${ }^{4}$, Robert Kieszko ${ }^{5}$, Janusz Milanowski ${ }^{5}$, Daria Świniuch ${ }^{6}$, Rodryg Ramlau ${ }^{6}$, Ewa Chmielowska ${ }^{7,8}$, Maciej Krzakowski ${ }^{1}$

'Department of Lung Cancer and Chest Tumors, Maria Sklodowska-Curie National Research Institute of Oncology, Warsaw, Poland 2Department of Oncology, The John Paul II Specialist Hospital, Kraków, Poland

${ }^{3}$ Oncology Department, E.J. Zeyland Wielkopolska Center of Pulmonology and Thoracic Surgery, Poznan, Poland

${ }^{4}$ Department of Oncology and Radiotherapy, Medical University of Gdansk, Gdansk, Poland

${ }^{5}$ Department of Pneumonology, Oncology and Allergology, Medical University of Lublin, Lublin, Poland

${ }^{6}$ Department of Oncology, Poznan University of Medical Scienses, Poznan, Poland

${ }^{7}$ Department of Oncology, Oncology Centre, Bydgoszcz, Poland

${ }^{8}$ Clinical Oncology Department, Oncology Hospital Tomaszow Mazowiecki, Poland

\title{
Effectiveness of osimertinih in patients with lung adenocarcinoma in clinical practice — the Expanded Drug Access Program in Poland
}

Abstract

Introduction: 0simertinib is a third-generation, irreversible epidermal growth factor receptor (EGFR) tyrosine kinase inhibitor that has demonstrated efficacy in the treatment of EGFR-mutant non-small-cell lung cancer (NSCLC) in prospective clinical trials. Material and methods: This retrospective analysis evaluated the outcomes of 32 pretreated patients with EGFR T790M mutation who received osimertinib in clinical practice at seven centers in Poland within the Expanded Drug Access Program. Osimertinib was used in the second line in $59 \%$ of patients and in later lines in $41 \%$.

Results: Objective response was attained in 16 patients $(50 \%)$, and 12 subjects (38\%) had stable disease. Median progression -free survival was 11.3 months in the overall population, 12.6 months in patients with EGFR exon 19 mutation and 7.5 months in patients with EGFR exon 21 mutation ( $p=0.045$ ). Median overall survival (OS) was 18.3 months. Overall, $58.4 \%$ and $45.6 \%$ of patients remained in follow-up after 12 and 24 months, respectively. Median OS appeared longer for patients without cerebral metastases than for those with cerebral metastases (27.4 vs 9.4 months, respectively; $p=0.078$ ), and for patients with the Eastern Cooperative Oncology Group performance status (ECOG PS) 0-1 than those with ECOG PS 2 (27.4 vs 11.8 months, respectively; $p=0.189$ ), although neither result reached statistical significance. Median OS of patients with partial response, stable disease and progressive disease was $27.4,12.7$ and 4.5 months, respectively $(p<0.001)$. Age, comorbidities, line of treatment with osimertinib, and type of activating EGFR mutation did not impact on OS. Adverse events of any grade or grade $3 / 4$ were reported in $38 \%$ and $9 \%$ of patients, respectively. One person discontinued due to interstitial pneumonia.

Conclusion: These results confirm the value of osimertinib in patients with previously treated EGFR T790M-mutant NSCLC. Clinical benefit was evident in patients with cerebral metastases and moderate performance status.

Key words: non-small-cell lung cancer, epidermal growth factor receptor tyrosine kinase inhibitor, T790M mutation, osimertinib, clinical practice

Adv Respir Med. 2020; 88: 189-196

\section{Introduction}

Advanced non-small-cell lung cancer (NSCLC) remains a disease of poor prognosis. The effectiveness of chemotherapy is limited: overall response rates (ORRs) do not exceed $25-30 \%$, and median overall survival (OS) is $10-12$ months [1]. Patients whose tumors harbor activating mutations in the epidermal growth factor receptor (EGFR) gene - most of whom have adenocarcinoma - comprise a clinically important subgroup. The prevalence of EGFR mutation varies accor-

Address for correspondence: Magdalena Knetki-Wróblewska, Department of Lung Cancer and Chest Tumors, Maria Skłodowska-Curie Memorial Cancer Center, Warsaw, Poland; e-mail: magdalena.knetki@coi.waw.pl

DOI: 10.5603/ARM.2020.0130

Received: 14.11 .2019

Copyright (C) 2020 PTChP

ISSN 2451-4934 
ding to ethnicity and is estimated at $30-45 \%$ in Asian patients and 10-20\% in Caucasian patients [1]. The prognosis of this molecularly selected subgroup of patients has significantly improved with the introduction of EGFR tyrosine kinase inhibitor (TKI) drugs. In first-line treatment, erlotinib, gefitinib or afatinib provide an ORR of approximately $60 \%$ and a median progression-free survival (PFS) of around 10-12 months [2-4], while dacomitinib and osimertinib yield a median PFS of approximately 18 months [5, 6].

Until recently, the treatment of choice for disease progression during EGFR TKI therapy was chemotherapy with platinum-based drugs. However, identification of the molecular mechanisms responsible for resistance to anti-EGFR treatment has allowed the development of novel drugs targeting resistant tumors. Currently, osimertinib is the only agent approved for use in this setting.

Osimertinib is a third-generation, irreversible EGFR TKI. It has demonstrated significant benefit vs platinum-based chemotherapy after failure of first- or second-generation EGFR TKIs in patients whose tumors harbor the T790M resistance mutation, of whom $60 \%$ showed primary TKI sensitivity [7, 8]. Furthermore, osimertinib is also characterized by high activity in the first-line treatment of subjects with activating EGFR mutation-positive tumors [6].

This paper reports the outcomes of osimertinib treatment in patients with advanced NSCLC with confirmed EGFR T790M mutation after disease progression during EGFR TKI therapy. Treatment was carried out as a part of the Expanded Drug Access Program prior to reimbursement of the osimertinib in Poland.

\section{Material and methods}

\section{Eligibility criteria}

The study included 32 patients who qualified for treatment with osimertinib as a part of the Expanded Drug Access Program at seven cancer centers in Poland between July 2016 and March 2018. Eligible patients had a diagnosis of advanced NSCLC at stage IV or stage IIIB (with no radical treatment options), confirmed EGFR T790M mutation, measurable lesions, age > 18 years, Eastern Cooperative Oncology Group performance status (ECOG PS) 0-2, normal laboratory indicators of renal, hepatic and hematopoietic function, and absence of clinically active secondary central nervous system (CNS) lesions. Patients with a history of interstitial lung disease, radiation pneumonitis requiring glucocorticosteroid use, electrocardiogram abnormalities or risk factors for QT prolongation were not eligible for treatment.

Osimertinib was administered at a daily dose of $80 \mathrm{mg}$ until progressive disease (PD) was confirmed, unacceptable toxicity occurred, or a patient's consent was withdrawn. Response assessment was based on computed tomography (CT) performed approximately every 8 weeks.

Before starting the treatment, patients signed an informed consent form for osimertinib therapy.

\section{Study objectives and outcomes}

The aim of the study was to assess the efficacy and safety of osimertinib in patients diagnosed with advanced NSCLC and T790M mutation after failure of previous systemic treatment. Treatment outcomes were retrospectively evaluated using available medical documentation. Radiological images were not reassessed for the purposes of this study.

Response was assessed according to Response Evaluation Criteria in Solid Tumors v. 1.1. In addition, PFS was evaluated as the duration from osimertinib initiation until documented radiological/clinical disease progression or death. OS was defined as the duration from the initiation of osimertinib until death. The study also assessed the prognostic value of selected clinical and molecular factors: age; gender; ECOG PS; smoking status; previous cancer treatment; presence of cerebral metastases; type of EGFR activating mutation; and response to previous treatment. The safety of the drug was analyzed by evaluation of adverse reactions, which were classified according to the Common Terminology Criteria for Adverse Events v. 4.0.

\section{Statistical methods}

PFS and OS were analyzed using Kaplan-Meier estimators. Results are presented as median survival and 12- and 24-month survival rates. The log-rank test was used to compare PFS and OS between subgroups defined basing on the analyzed clinical and molecular variables. A significance level (alpha) of 0.05 was applied in all calculations. Analyses were conducted using the R statistical program v. 3.5.1 (2018; R Foundation for Statistical Computing, Vienna, Austria).

\section{Results}

\section{Patient population}

A total of 32 patients were included in the study. At the time of the analysis, 15 subjects 
remained in follow-up, of whom 7 persons continued to receive osimertinib. The median duration of follow-up since the initiation of the treatment was 40.9 months.

Table 1 summarizes key patient characteristics at the start of osimertinib therapy. Overall, 15 patients (47\%) had never smoked, while 12 subjects $(38 \%)$ were past smokers and 2 individuals (6\%) were current smokers. A total of 17 patients (53\%) had documented comorbidities, the most common of which was hypertension (11 patients; 34\%). All patients subjects had a diagnosis of lung adenocarcinoma. Twenty patients $(63 \%)$ had stage IV disease at diagnosis, while the remaining patients ( $\mathrm{n}=12 ; 38 \%)$ were diagnosed at stages I-IIIB and had previously received treatment with radical intent prior to diagnosis of advanced disease. Between initial diagnosis and eligibility for the osimertinib treatment, 8 patients (25\%) had secondary changes within the CNS for which they had received palliative

Table 1. Patient characteristics at the initiation of osimertinib

\begin{tabular}{|c|c|}
\hline & Patients $(\mathrm{n}=\mathbf{3 2}$ ) \\
\hline Median age (range), years & $64(37-82)$ \\
\hline \multicolumn{2}{|l|}{ Gender, n (\%) } \\
\hline Male & $13(41)$ \\
\hline Female & $19(59)$ \\
\hline \multicolumn{2}{|l|}{ ECOG PS, n (\%) } \\
\hline 0 & $3(9)$ \\
\hline 1 & $19(59)$ \\
\hline 2 & $10(31)$ \\
\hline \multicolumn{2}{|c|}{ Type of EGFR activating mutation, n (\%) } \\
\hline Del 19 & $22(69)$ \\
\hline L858R & $8(25)$ \\
\hline Other & $1(3)$ \\
\hline \multicolumn{2}{|l|}{ Smoking history, n (\%) } \\
\hline Never & $15(47)$ \\
\hline Past smoker & $12(38)$ \\
\hline Current smoker & $2(6)$ \\
\hline Missing & $3(9)$ \\
\hline \multicolumn{2}{|c|}{ Location of metastatic lesions, $\mathbf{n}(\%)$} \\
\hline Lungs & $17(53)$ \\
\hline CNS & $8(25)$ \\
\hline Liver & $6(19)$ \\
\hline Bone & $8(25)$ \\
\hline Adrenal glands & $2(6)$ \\
\hline \multicolumn{2}{|c|}{ Number of prior treatment lines, $\mathbf{n}(\%)$} \\
\hline 1 & $19(59)$ \\
\hline 2 & $5(16)$ \\
\hline 3 & $5(16)$ \\
\hline 4 & $2(6)$ \\
\hline
\end{tabular}

CNS - central nervous system; Del 19 - exon 19 deletion; ECOG PS Eastern Cooperative Oncology Group performance status; EGFR — epidermal growth factor receptor brain irradiation.

\section{Previous treatment}

All study subjects had received systemic treatment prior to the initiation of osimertinib. Most patients (59\%) had received one line of prior systemic treatment with a first- or second-generation EGFR TKI (erlotinib, 53\%; afatinib, 33\%; gefitinib, 10\%; dacomitinib, 3\%). The most common types of activating mutation in the EGFR gene were exon 19 deletions (71\%). Among 30 patients with documented response assessments during prior EGFR TKI therapy, 25 individuals (83\%) had achieved response to treatment. After EGFR TKI treatment, 13 patients (40\%) received chemotherapy, which mostly consisted of platinum-containing regimens. Liquid biopsy was used to confirm the presence of the T790M mutation in most cases (27 patients; $84 \%$ ).

\section{Effectiveness}

Response. Response to the treatment was evaluated in all 32 study subjects (Table 2). Objective response was documented in 16 patients (50\%), while 12 patients (38\%) attained stable disease (SD). A total of 28 patients (88\%) therefore obtained clinical benefit. In 4 patients (13\%), progressive disease (PD) was reported as the best response to treatment. Among the 8 patients with CNS lesions, 1 individual (13\%) achieved complete regression of lesions, 4 patients had partial response (PR; 50\%) and 3 patients had SD (38\%) within the CNS.

There was no effect of selected clinical/molecular factors (age, smoking status, ECOG PS, and type of activating EGFR mutation) on the ORR to osimertinib treatment.

Of the 25 patients who discontinued osimertinib, PD data were available in 23 cases. The most frequent sites of PD were the lungs (50\%)

Table 2. Response to treatment with osimertinib

\begin{tabular}{lc}
\hline & Patients, $\mathbf{n}(\%)$ \\
\hline Overall response $(\mathbf{n}=\mathbf{3 2})$ & \\
PR & $16(50)$ \\
SD & $12(38)$ \\
PD & $4(13)$ \\
\hline Response of CNS lesions $(\mathbf{n}=\mathbf{8})$ & \\
CR & $1(13)$ \\
PR & $4(50)$ \\
SD & $3(38)$ \\
\hline
\end{tabular}

CR — complete response; PD — progressive disease; PR — partial response; $\mathrm{SD}$ — stable disease 
and liver (15\%). Progression within the CNS was observed in 2 patients (including 1 individual with symptomatic brain metastases who had previously received radiotherapy to the brain). After PD, 6 patients (24\%) received another line of systemic treatment, while 13 subjects (44\%) received only best supportive care or palliative irradiation. Data on post-progression therapy were unavailable for the remaining 6 patients.

Progression-free survival. At the time of the analysis, 15 patients (47\%) were still alive and 7 patients (22\%) were still receiving osimertinib. Median PFS was 11.3 months. The proportions of patients who remained alive and without signs of PD were $49.2 \%$ at 12 months and $17.0 \%$ at 24 months.

Age, comorbidities, osimertinib treatment line, and type of prior EGFR TKI therapy had no impact on PFS. However, a correlation between PFS and the type of activating EGFR mutation was shown: median PFS was 12.6 months in patients with exon 19 deletions, compared with 7.5 months in those with exon 21 point mutations ( $p=0.045$; Figure 1). The prognostic value of the type of activating mutation was also shown in terms of PFS rates at 12 months (exon 19 deletions, 54\%; exon 21 point mutations, 17\%) and 24 months (exon 19 deletions, 14\%; exon 21 point mutations, 0\%).

In addition, response to prior first- and second-generation EGFR TKI treatment also demonstrated prognostic value (Figure 2). In patients with objective response to prior treatment,

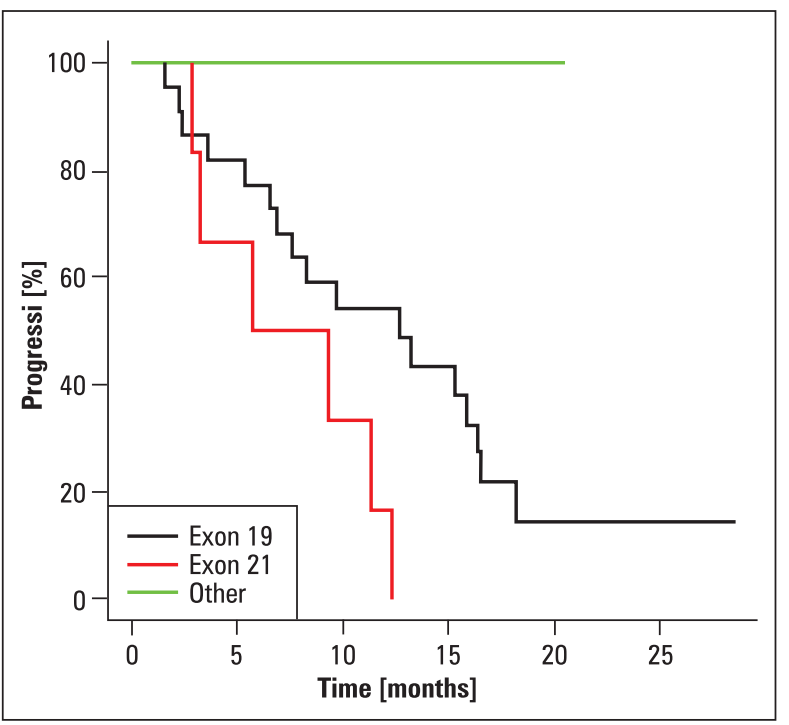

Figure 1. PFS curves according to type of activating EGFR mutation. Del 19 - exon 19 deletion; EGFR - epidermal growth factor receptor; Point 21 - exon 21 point mutation; PFS — progression-free survival median PFS during osimertinib treatment was 12.6 months. Two patients with PD as the best response to previous EGFR TKI therapy did not benefit from osimertinib: PD was documented in the first CT examination performed 2 months after the start of therapy ( $\mathrm{p}<0.001$ ).

Overall survival. Median OS was 18.3 months. The percentage of patients who remained in follow-up after 12 and 24 months of therapy was $58.4 \%$ and $45.6 \%$, respectively. Age, comorbidities, osimertinib treatment line, and type of activating EGFR mutation had no effect on OS. However, response to osimertinib demonstrated prognostic value: median OS was 27.4 months in the subjects with PR, 12.7 months in patients with SD, and 4.5 months in patients with $\mathrm{PD}$ as the best response to treatment ( $p<0.001$; Figure 3 ).

Response to the prior first- and second-generation EGFR TKI treatment also had a significant prognostic impact on OS following the osimertinib treatment. Median OS was 18.3 months in patients with objective response to prior treatment $(\mathrm{n}=25)$, whereas the 2 individuals with PD during previous anti-EGFR treatment had OS times of 2.5 and 4.0 months ( $p<0.001$; Figure 4$)$.

The impact of ECOG PS at the time of qualification for osimertinib on survival rates was also analyzed. Patients with very good and good performance score (ECOG PS 0-1) had longer median OS (27.4 months) than those with moderate performance score (ECOG PS 2; 11.8 months). However, the observed difference was not stati-

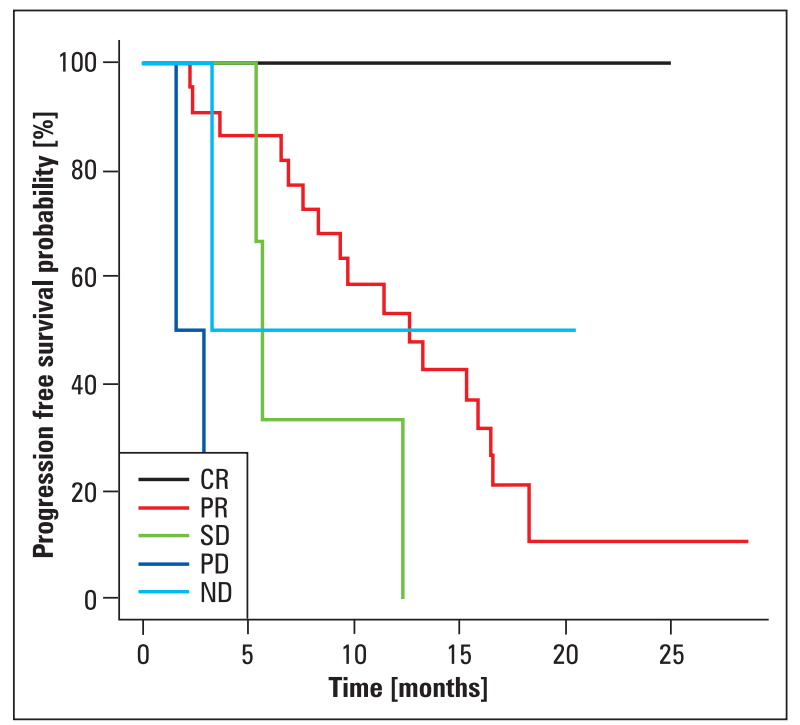

Figure 2. PFS curves according to response to prior TKI therapy. CR complete response; $\mathrm{PD}$ — progressive disease; PFS — progression-free survival; PR — partial response; SD — stable disease; ND — no data 


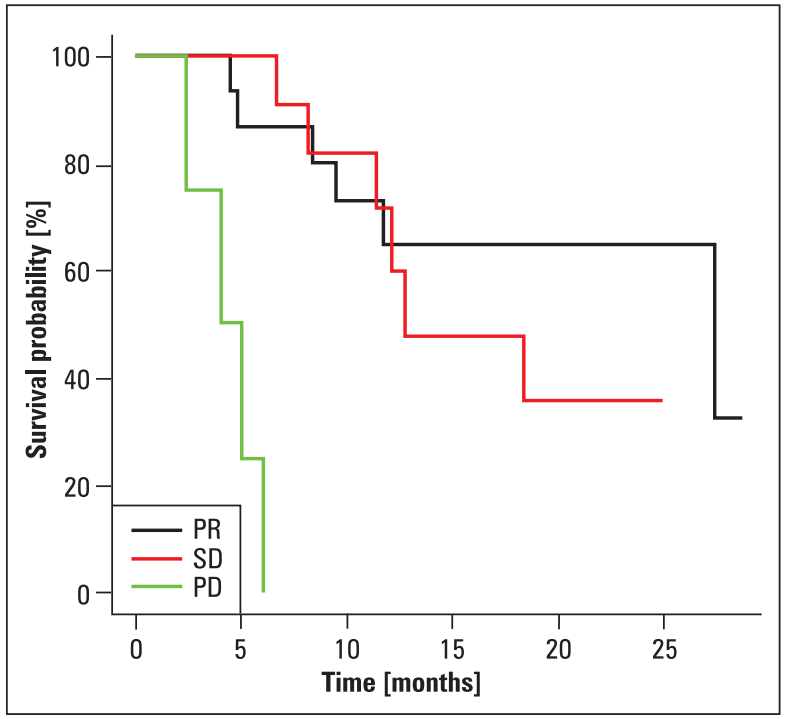

Figure 3. OS curves according to response to osimertinib. OS overall survival; PD — progressive disease; PR — partial response; $\mathrm{SD}$ - stable disease

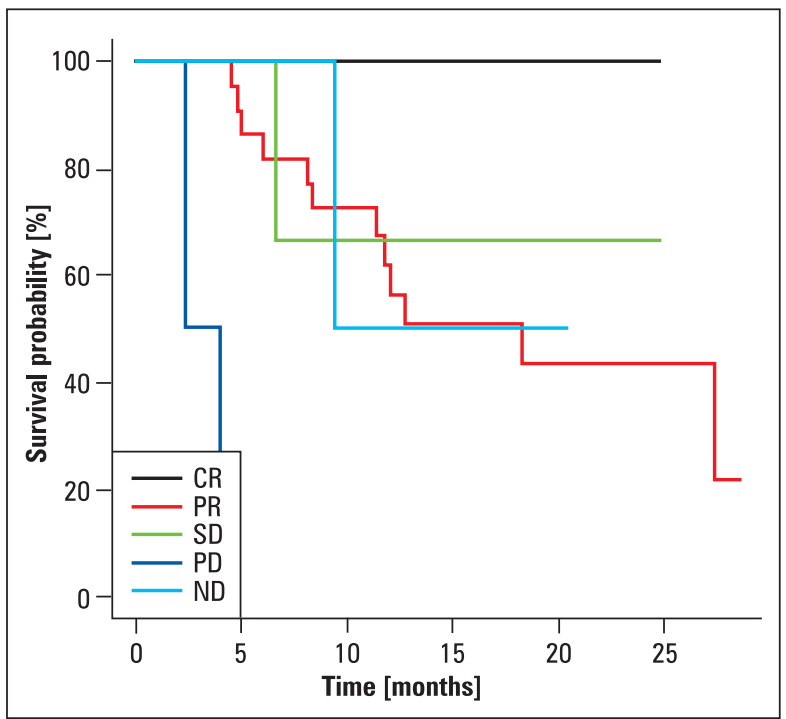

Figure 4. OS curves according to response to previous first- or secondgeneration EGFR TKIs. CR — complete response; EGFR — epidermal growth factor receptor; $\mathrm{OS}$ - overall survival; $\mathrm{PD}$ - progressive disease; PR — partial response; SD — stable disease; TKI — tyrosine kinase inhibitor

stically significant ( $p=0.159$; Figure 5$)$.

The presence of cerebral metastases also impacted on the course of the disease. Patients without secondary brain lesions lived longer than those with documented brain lesions, with median OS of 27.4 and 9.4 months, respectively $(\mathrm{p}=0.078$; Figure 6).

Due to the small size of the patient population, no multifactorial analysis was performed.

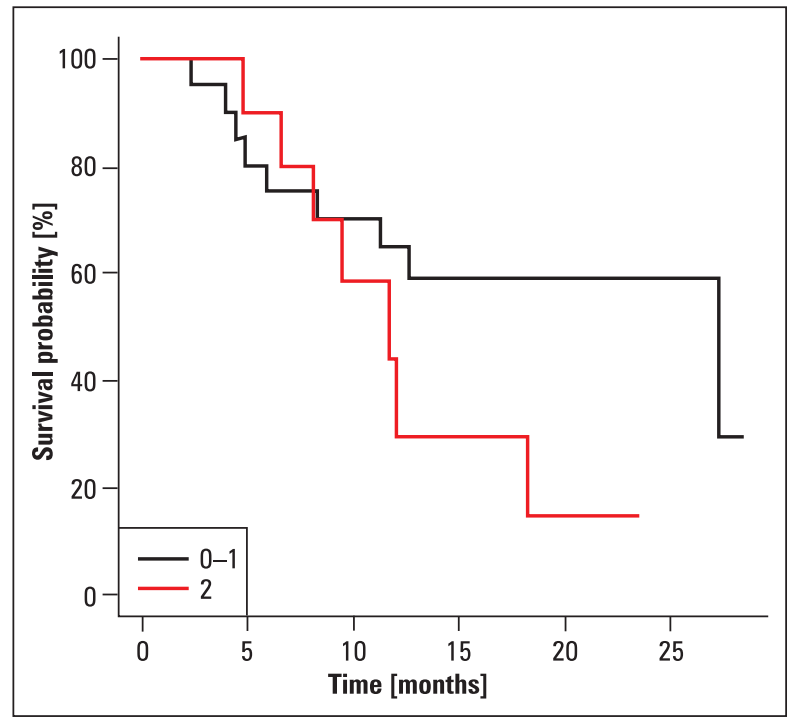

Figure 5. OS curves according to ECOG PS. ECOG PS - Eastern Cooperative Oncology Group performance status; OS — overall survival

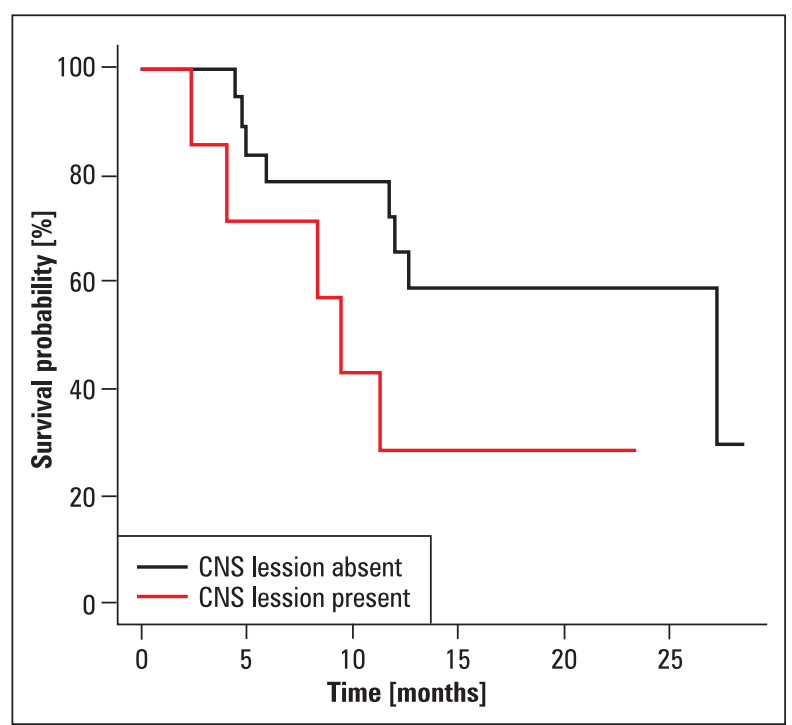

Figure 6. Overall survival (OS) curves according to the presence of secondary lesions in the CNS

Univariate analyses assessing the prognostic value of selected clinical and molecular factors in terms of OS are summarized in Table 3. Survival parameters for the overall group are presented in Table 4.

\section{Safety}

Adverse events were reported at any grade for 12 patients (38\%) and at grade $3 / 4$ for 3 patients (9\%; Table 5). In total, 6 study subjects (18\%) had skin lesions and 3 patients (9\%) had diarrhea. Other adverse events each occurred in 


\section{Table 3. The prognostic value of selected parameters in terms of OS}

\begin{tabular}{|c|c|c|}
\hline & Median OS, months & P-value \\
\hline ECOG PS: 0-1 vs 2 & 27.4 vs 11.8 & 0.189 \\
\hline Age: $<60$ years $v s \geq 60$ years & 27.4 vs 12.7 & 0.686 \\
\hline CNS metastases: present $v s$ absent & 27.4 vs 9.4 & 0.078 \\
\hline Bone metastases: present vs absent & 27.4 vs 11.9 & 0.089 \\
\hline Liver metastases: present $v s$ absent & 12.1 vs 9.4 & 0.160 \\
\hline Response to osimertinib: CR/PR vs PD & 27.4 vs 4.5 & $<0.001$ \\
\hline Response to prior first- or second-generation EGFR TKIs: CR/PR vs PD & 18.3 vs 3.2 & $<0.001$ \\
\hline Type of EGFR mutation: Del 19 vs L858R & 18.3 vs 12.1 & 0.379 \\
\hline Line of osimertinib treatment: second vs third or later & 27.4 vs 9.9 & 0.088 \\
\hline
\end{tabular}

CR — complete response; ECOG PS — Eastern Cooperative Oncology Group performance status; EGFR — epidermal growth factor receptor; OS — overall survival; $\mathrm{PD}$ - progressive disease; PR — partial response; TKI — tyrosine kinase inhibitor

Table 4. PFS and OS in the analyzed group of patients

\begin{tabular}{lccc}
\hline & Median, months & 12-month rate, \% & 24-month rate, \% \\
\hline PFS & 11.3 & 49.2 & 17.0 \\
OS & 18.3 & 58.4 & 45.6 \\
\hline
\end{tabular}

OS — overall survival; PFS — progression-free survival

Table 5. Adverse reactions to osimertinib $(n=32)$

\begin{tabular}{lcc}
\hline & \multicolumn{2}{c}{ Patients, $\mathbf{n}(\%)$} \\
\cline { 2 - 3 } & Any grade & Grade 3/4 \\
\hline All adverse events & $12(38)$ & $3(9)$ \\
Skin lesions & $6(19)$ & - \\
Diarrhea & $3(9)$ & $2(6)$ \\
Interstitial pneumonia & $1(3)$ & $1(3)$ \\
Elevated activity of liver enzymes & $1(3)$ & - \\
\hline
\end{tabular}

1 individual [interstitial pneumonia, elevated activity of liver enzymes, and "other" (no further data available)]. Clinically significant side effects included interstitial pneumonia (1 patient) and diarrhea (2 patients). In 4 subjects (13\%), adverse events led to treatment interruption. The patient with interstitial pneumonia associated with osimertinib permanently discontinued treatment. There were no treatment-related deaths.

\section{Discussion}

This report describes treatment outcomes with osimertinib in 32 patients diagnosed with advanced lung adenocarcinoma and documented activating T790M EGFR mutation after failure of first- or second-generation EGFR TKI therapy. Treatment was carried out in routine clinical practice in the general patient population and not as part of a clinical trial. At the time of the analysis, 15 patients (47\%) were still alive and 7 patients (22\%) were still receiving osimertinib.

Clinical benefit was observed in $88 \%$ of the study subjects and PR in $50 \%$ of patients. The ORR is slightly lower than in the AURA2 study and other reports [6-8], which could be explained by less precise assessment of CT results in clinical practice compared with clinical trials — ORR was evaluated basing on medical documentation and less strict qualification to EAP comparing to 
the clinical trials. The very high proportion of patients with clinical benefit is consistent with other reports and confirms the efficacy of osimertinib in patients with T790M-positive NSCLC. No correlation was found between treatment response and the clinical factors analyzed. In case of the patients with documented PD as the best response to osimertinib, the presence of other concomitant mechanisms of resistance to TKI EGFR treatment, apart from the T790M mutation, should be considered [9].

In the analyzed group, a relationship was demonstrated between the type of activating mutation in the EGFR gene found initially and the duration of response to osimertinib. More favorable outcomes were observed in patients with exon 19 deletions than in those with exon 21 point mutations (median PFS, 12.6 vs 7.5 months, respectively). Recently published, updated results from patients treated with osimertinib in two phase II studies also showed the type of activating EGFR mutation to be a prognostic factor [10]. In that analysis, patients with exon 19 deletions derived a greater benefit than individuals with exon 21 mutation in terms of ORR (70\% vs 57\%), median PFS (11.1 vs 9.5 months) and median OS (29.1 vs 21.4 months) [10]. The current study also provided some evidence that the type of activating mutation impacted on OS, although the difference was not statistically significant. In another analysis based on a larger group of osimertinib-treated patients in clinical practice, median OS was significantly longer in subjects with exon 19 deletions than in patients with exon 21 mutations (23.1 vs 15.3 months; $p$ $=0.03)[11]$.

We observed a relationship between response to osimertinib and OS in the present study. Similar conclusions have been presented from an analysis of 27 patients treated in clinical practice [12]. The median OS of patients who achieved objective response to treatment was 24.2 months vs 13.5 months in the group of patients with SD or PD.

In the current study, 10 patients (31\%) had ECOG PS 2 at the time of qualification for osimertinib treatment. The collecting of data on the efficacy and safety of the drug in this group of patients is particularly important, because only individuals with ECOG PS 0 or 1 are usually qualified for therapy in the clinical trials. Poor performance status seems to be a negative prognostic factor in patients receiving EGFR TKIs. In our dataset, median OS appeared longer in patients with ECOG PS 0-1 than in those with ECOG PS
2 (27.4 vs 11.8 months, respectively), although the difference was not statistically significant. Data from the GIOTAG observational study, which evaluated sequential treatment with afatinib and osimertinib in 204 patients, also indicate that worse performance status is associated with poorer outcomes [13]. Median duration of the sequential treatment strategy was 31.3 months in patients with ECOG $0-1$ and 22.2 months in patients with ECOG $\geq 2$ ( $p<0.001$ ) [13]. Recently, results of osimertinib treatment in a group of 30 patients with moderate performance status (ECOG 2-3) have been published, in which 53\% of patients attained objective responses to treatment and median PFS was 8.2 months [14]. The majority of the subjects (63\%) had an improvement in their general condition and the rates of adverse reactions and treatment-related deaths were in line with expectations based on other reports in the literature. In a separate analysis of the efficacy and safety of osimertinib in a group of 12 patients (out of 51 in total) with a moderate performance status (ECOG 2-3), the prognostic value of patient fitness was not specifically evaluated, but the authors emphasized that almost all subjects with ECOG 2-3 had clinical benefit from treatment [15].

Cerebral metastases are a significant clinical problem in EGFR mutation-positive NSCLC that are estimated to affect approximately $20 \%$ of patients at diagnosis and almost $60 \%$ at some point during the course of their disease [16]. The treatment of choice is whole-brain radiotherapy (WBRT) or, if possible, local treatment using stereotactic radiotherapy or surgery. Due to its high CNS penetration, osimertinib could be a relevant treatment option in this setting [17]. Supporting clinical data are provided by the FLAURA study of first-line osimertinib in a subgroup analysis of patients with CNS lesions at baseline. In that subgroup, osimertinib was associated with an ORR in the CNS of $66 \%$ (compared with $43 \%$ with gefitinib) [18]. Osimertinib also significantly reduced the risk of progression of CNS lesions (median CNS PFS not reached with osimertinib vs 13.8 months with gefitinib; hazard ratio, 0.48). In that study, brain imaging was not mandatory for all patients and was performed only in those with clinical suspicion of secondary CNS lesions. Only $25 \%$ of patients with confirmed CNS lesions had previous radiotherapy to the CNS; the remaining subjects were qualified for the osimertinib treatment.

In the Expanded Access Drug Program, patients with CNS lesions are required to receive 
local treatment and demonstrate complete control of symptoms to be eligible for osimertinib and other EGFR TKIs. In the current study, CNS were identified at some point after diagnosis in 8 patients, all of whom had prior local treatment (WBRT) and derived clinical benefit from subsequent osimertinib therapy. However, it should be emphasized that CNS imaging was not mandatory and the data presented may not be complete.

\section{Summary}

The present study confirms the efficacy and safety of osimertinib in patients with NSCLC after EGFR TKI failure. Clinical benefit was obtained by patients with the T790M mutation in both the second and subsequent lines of treatment, as well as in the subjects with secondary CNS lesions and moderate performance status. The type of activating mutation present at initial diagnosis showed prognostic value for PFS, while response to osimertinib treatment and response to prior TKI therapy both had an effect on OS.

\section{Conflict of interest}

MKW: lectures: Astra-Zeneca, Roche, Boehringer-Ingelheim, Pfizer, MSD, BMS.

DMK: advisory board and consultancy: Roche, Boehringer-Ingelheim, AstraZeneca, MSD, BMS, TAKEDA, Pfizer, MERCK.

MB: advisory board and lectures : Roche, Boehringer-Ingelheim, AstraZeneca, MSD, BMS, Pfizer.

AW: travel grants and lectures: BMS, Roche, Pfizer, Takeda.

RD: advisory board and honoraria: AstraZeneca, Roche, Boehringer-Ingelheim, Pfizer, Novartis, MSD, Seattle Genetics, Foundation Medicine.

RR: advisory board: Boehringer-Ingelheim, Roche, Takeda, MSD, Pfizer, Novartis, BMS, Abbvie.

\section{References:}

1. Planchard D, Popat S, Kerr K, et al. Metastatic non-small cell lung cancer: ESMO Clinical Practice Guidelines for diagnosis, treatment and follow-up. Annals of Oncology. 2018; 29: iv192iv237, doi: 10.1093/annonc/mdy275.

2. Sequist L, Yang JH, Yamamoto N, et al. Phase III study of afatinib or cisplatin plus pemetrexed in patients with metastatic lung adenocarcinoma with EGFR mutations. Journal of Clinical Oncology. 2013; 31(27): 3327-3334, doi: 10.1200/jco.2012.44.2806.

3. Rosell R, Carcereny E, Gervais R, et al. Erlotinib versus standard chemotherapy as first-line treatment for European patients with advanced EGFR mutation-positive non-small-cell lung cancer (EURTAC): a multicentre, open-label, randomised phase 3 trial. Lancet Oncol. 2012; 13: 239-246.
4. Mok T, Wu YL, Thongprasert S, et al. Gefitinib or carboplatin-paclitaxel in pulmonary adenocarcinoma. New England Journal of Medicine. 2009; 361(10): 947-957, doi: 10.1056/ nejmoa0810699.

5. Wu YL, Cheng Y, Zhou X, et al. Dacomitinib versus gefitinib as first-line treatment for patients with EGFR-mutation-positive non-small-cell lung cancer (ARCHER 1050): a randomised, open-label, phase 3 trial. The Lancet Oncology. 2017; 18(11): 1454-1466, doi: 10.1016/s1470-2045(17)30608-3.

6. Soria JC, Ohe Y, Vansteenkiste J, et al. Osimertinib in untreated EGFR-mutated advanced non-small-cell lung cancer. New England Journal of Medicine. 2018; 378(2): 113-125, doi: 10.1056/nejmoa1713137.

7. Mok T, Wu YL, Ahn MJ, et al. Osimertinib or platinum-pemetrexed in EGFR t790m-positive lung cancer. New England Journal of Medicine. 2017; 376(7): 629-640, doi: 10.1056/nejmoa1612674.

8. Goss G, Tsai CM, Shepherd F, et al. Osimertinib for pretreated EGFR Thr790Met-positive advanced non-small-cell lung cancer (AURA2): a multicentre, open-label, single-arm, phase 2 study. The Lancet Oncology. 2016; 17(12): 1643-1652, doi: 10.1016/s1470-2045(16)30508-3.

9. Chen M, Xu Y, Zhao J, et al. Concurrent driver gene mutations as negative predictive factors in epidermal growth factor receptor-positive non-small cell lung cancer. EBioMedicine. 2019; 42: 304-310, doi: 10.1016/j.ebiom.2019.03.023.

10. Ahn M, Tsai C, Shepherd F, et al. Osimertinib in patients with T790M mutation-positive, advanced non-small cell lung cancer: Long-term follow-up from a pooled analysis of 2 phase 2 studies. Cancer. 2018; 125(6): 892-901, doi: 10.1002/ cncr.31891.

11. Auliac J, Pérol M, Planchard D, et al. Real-life efficacy of osimertinib in pretreated patients with advanced non-small cell lung cancer harboring EGFR T790M mutation. Lung Cancer. 2019; 127: 96-102, doi: 10.1016/j.lungcan.2018.11.037.

12. Yoshimura A, Yamada T, Okura N, et al. Clinical characteristics of osimertinib responder in non-small cell lung cancer patients with EGFR-T790M mutation. Cancers. 2019; 11(3): 365, doi: 10.3390/cancers11030365.

13. Hochmair M, Morabito A, Hao D, et al. Sequential treatment with afatinib and osimertinib in patients with EGFR mutation -positive non-small-cell lung cancer: an observational study. Future Oncology. 2018; 14(27): 2861-2874, doi: 10.2217/fon2018-0711.

14. Nakashima K, Kimura M, Akamatsu H, et al. Osimertinib for patients with EGFR T790M mutation-positive non-small-cell lung cancer and a poor performance status. Japanese Journal of Clinical Oncology. 2019; 49(7): 671-675, doi: 10.1093/jjco/ hyz041.

15. Stratmann J, Michels S, Hornetz S, et al. Efficacy and safety analysis of the German expanded access program of osimertinib in patients with advanced, T790M-positive non-small cell lung cancer. Journal of Cancer Research and Clinical Oncology. 2018; 144(12): 2457-2463, doi: 10.1007/s00432-018-2754-x.

16. Wrona A, Dziadziuszko R, Jassem J. Management of brain metastases in non-small cell lung cancer in the era of tyrosine kinase inhibitors. Cancer Treatment Reviews. 2018; 71: 59-67, doi: 10.1016/j.ctrv.2018.10.011.

17. Ballard P, Yates J, Yang Z, et al. Preclinical comparison of osimertinib with other EGFR-TKIs in EGFR-mutant NSCLC brain metastases models, and early evidence of clinical brain metastases activity. Clinical Cancer Research. 2016; 22(20): 5130-5140, doi: 10.1158/1078-0432.ccr-16-0399.

18. Reungwetwattana T, Nakagawa K, Cho B, et al. CNS response to osimertinib versus standard epidermal growth factor receptor tyrosine kinase inhibitors in patients with untreated EGFR-mutated advanced non-small-cell lung cancer. Journal of Clinical Oncology. 2018; 36(33): 3290-3297, doi: $\underline{10.1200 /}$ jco.2018.78.3118.

19. Goss G, Tsai CM, Shepherd FA, et al. CNS response to osimertinib in patients with T790M-positive advanced NSCLC: pooled data from two phase II trials. Annals of Oncology. 2018; 29(3): 687-693, doi: 10.1093/annonc/mdx820. 